Proceedings of the 43rd "Jaszowiec", International School and Conference on the Physics of Semiconductors, Wisła 2014

\title{
Magnetic Field Effect on the Excitation Spectrum of a Neutral Exciton in a Single Quantum Dot
}

\begin{abstract}
M.R. Molas ${ }^{a, b, *}$, A.A.L. Nicolet ${ }^{b}$, B. PiętKA ${ }^{a}$, A. BABiński ${ }^{a}$ AND M. Potemski ${ }^{b}$
${ }^{a}$ Faculty of Physics, University of Warsaw, L. Pasteura 5, 02-093 Warszawa, Poland

${ }^{b}$ Laboratoire National des Champs Magnétiques Intenses, CNRS-UJF-UPS-INSA, 25, av. des Martyrs, 38042 Grenoble, France

Excitation-energy-dependent magnetospectroscopic measurements of a single GaAlAs/AlAs quantum dot were performed. A significant effect of the excitation energy on the photoluminescence spectra is reported. The photoluminescence excitation spectroscopy has been used to investigate the excitation spectrum of a single electron-hole pair - a neutral exciton in magnetic field up to $14 \mathrm{~T}$. The observed resonances exhibit diamagnetic shift characteristic of an $s$-shell related emission. In our opinion, the creation of excited complexes involving an excited hole and a ground electron is responsible for the process.
\end{abstract}

DOI: 10.12693/APhysPolA.126.1066

PACS: 78.67.Hc, 71.35.-y, 78.55.Cr

\section{Introduction}

Semiconductor quantum dots (QDs) provide the confinement of carriers in all three dimensions of space at a scale comparable to their de Broglie wavelengths. This confinement leads to several effects which are of interest from both the fundamental and application points of view $[1,2]$. One of the appealing questions is the effect of electron-electron interactions on the excitation spectrum of the electron-hole $(\mathrm{e}-\mathrm{h})$ system strongly confined in a QD. It is known that these interactions lead to the mixing of quantum configurations, which affect the absorption spectra of $\mathrm{e}-\mathrm{h}$ complexes. The effect strongly depends on the number of confined electronic shells in the dot [3].

In order to study the excitations of an $\mathrm{e}-\mathrm{h}$ pair - a neutral exciton confined to a dot, the photoluminescence excitation (PLE) spectroscopy is often used [4]. Keeping in mind that in the PLE spectrum three processes (subsequent processes of absorption, relaxation, and recombination) are involved, the properly analysed PLE spectrum allows to resolve the absorption spectrum. The application of the magnetic field, which removes the orbital and spin degeneracy of excitonic states facilitates such an analysis.

In this communication we report on the optical properties of a single GaAlAs/AlAs QD. We investigate the photoluminescence (PL) and PLE spectra of the single dot in magnetic field up to $14 \mathrm{~T}$. The effect of the magnetic field on both types of the spectra is analysed. Moreover, the possible origin of the observed resonances of the neutral exciton is discussed.

\section{Experimental procedure}

The structure under investigation was grown using a molecular beam epitaxy. It contains GaAlAs QDs,

*corresponding author; e-mail: maciej.molas@fuw.edu.pl formed at the type II GaAs/AlAs heterostructure [5, 6]. Inside the dots, the GaAs/AlAs bilayer is replaced by $\mathrm{GaAs} / \mathrm{Ga}_{1-x} \mathrm{Al}_{x} \mathrm{As}$ material $(x<0.33)$, what results in lowering the potential energy and leads to a QD-type confinement. GaAlAs QDs have an extremely low surface density of about $10^{6} \mathrm{~cm}^{-2}$ with an estimated lateral diameter of about $50 \mathrm{~nm}$. Such a low surface density allows to address individual QDs without using e.g. metallic masks. A spectrally separated set of emission lines related to the single GaAlAs/AlAs QDs can be observed in the PL spectrum in a broad energy range (1.56$1.68 \mathrm{eV})$ [7-9].

The sample was placed on top of the $x-y-z$ piezo-stage in the bath cryostat and kept at $4.2 \mathrm{~K}$ in the helium exchange-gas [10]. The laser light, provided by a tuneable Ti:sapphire $\mathrm{cw}$ laser, was coupled to one branch of a Y-shaped fiber and focused by the microscope objective (spot size around $1 \mu \mathrm{m}^{2}$ )located directly above the sample. The signal was detected from the second branch of the fiber, by a $0.5 \mathrm{~m}$ monochromator equipped with a charge-coupled device camera cooled down with liquid nitrogen. The measurements have been carried out in the Faraday configuration with the aid of a superconducting magnet producing field up to $14 \mathrm{~T}$.

\section{Results \\ 3.1. Photoluminescence}

It has been found that the PL spectrum of a single GaAlAs/AlAs dot strongly depends on the power and the energy of the excitation -light. At low excitation powers in the non-resonant excitation regime (at the abovebarrier energy equal to $1.771 \mathrm{eV}$ ) three emission lines $\mathrm{X}$, $\mathrm{X}^{-}$, and $\mathrm{X}^{+}$occur - Fig. 1c. They were previously attributed to the neutral, negatively, and positively charged excitons, respectively $[9,11]$. At higher excitation powers - Fig. 1b, the $\mathrm{X}^{+}$line disappears from the spectrum -because of: the neutralization process of acceptors (the nominally undoped GaAs-based structures exhibit a residual $p$-type background doping) [12] and the asymmetry of the capture and the lifetime of electrons 
and holes (it is important in the non-resonant excitation regime) [11]. Moreover, an additional line, related to the doubly negatively charged exciton $\left(\mathrm{X}^{2-}\right)$, appears on the low-energy side of the $\mathrm{X}^{-}$line [7]. Further increase in the excitation power results in the emergence of emission lines in the energy range below the $\mathrm{X}^{2-}$ line, attributed to the recombination of multiexcitons (see Fig. 1c), and around $13 \mathrm{meV}$ above the $s$-shell emission, associated with the transition within the $p$-shell (not shown in the spectra).

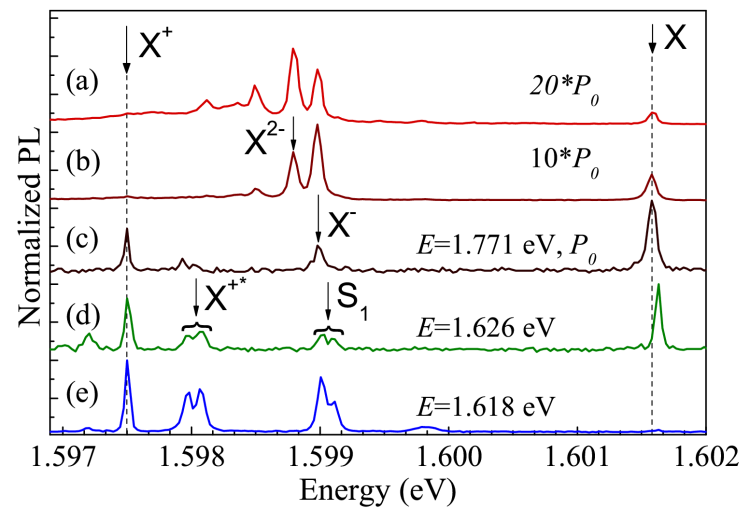

Fig. 1. PL spectra of a single GaAlAs QD excited nonresonantly at $E=1.771 \mathrm{eV}$ (a)-(c) with different excitation powers $\left(P_{0}\right)$ and resonantly at $E=1.626 \mathrm{eV}(\mathrm{d})$ or $E=1.618 \mathrm{eV}(\mathrm{e})$.

A different spectral pattern occurs- if the dot is excited resonantly (at the energy below the barrier: $1.626 \mathrm{eV}$ and $1.618 \mathrm{eV})$ - see Fig. 1d and e. The $\mathrm{X}$ and $\mathrm{X}^{+}$lines are accompanied by additional doublets: $\mathrm{X}^{+*}$, and $\mathrm{S}_{1}$. The $\mathrm{X}^{+*}$ line was previously identified as the emission of the excited positively charged exciton (also called a hot trion) [11]. The attribution of the $\mathrm{S}_{1}$ emission line is not clear, particularly as a result of its unexpected magnetic-field evolution described below. The lines related to the negatively charged excitons $\left(\mathrm{X}^{-}\right.$and $\left.\mathrm{X}^{2-}\right)$ are not present in the spectra. The difference between the PL spectra excited in the two excitation regimes reflects two distinct -mechanisms of carriers trapping in the single QD. The resonant excitation is a consequence of populating of the dot by e-h pairs, whereas the nonresonant by single carriers [13].

Moreover, the shift of the X energy - between the two excitation regimes appears - Fig. 1 - and it is analysed further in the paper.

The magnetic-field evolution of the spectra excited in the two excitation regimes is also studied (see Fig. 2). All the investigated emission lines split in the magnetic field. The $\mathrm{X}$ line splits into two components of opposite circular polarization, except for the lowest fields at which the exchange-interaction splitting can be observed [7]. The $\mathrm{X}^{+*}$ and $\mathrm{S}_{1}$ lines have double structure at $0 \mathrm{~T}$ and four-component patterns appear for both of them in the magnetic field. The $\mathrm{X}^{+*}$ structure originates from the electron-hole and hole-hole exchange interactions between holes occupying the $s$ - and $p$-shell levels

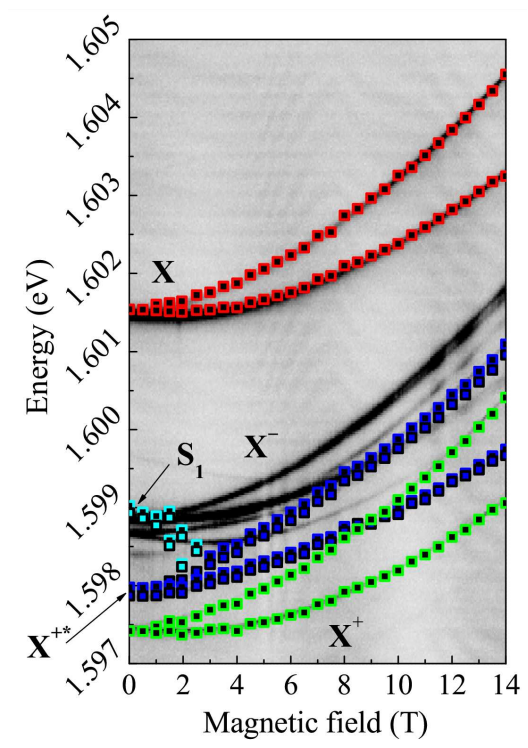

Fig. 2. The PL of a single GaAlAs QD as a function of magnetic field under two excitation regimes: the nonresonant - gray scale map, the resonant - color-points.

in the anisotropic lateral potential (for more details see Refs. $[11,14])$. Moreover, in the low-field range, the difference in the $\mathrm{X}$ emission energy between the two excitation regimes is observed. However, it disappears at magnetic field above $6 \mathrm{~T}$. That behaviour can be explained in our opinion by the competition between the Stark-shift effect attributed to the neutralization of acceptors and the effect of an additional confinement produced by the magnetic field. At low fields, the influence of the latter one can be neglected in comparison with the Stark shift. In the high-field regime, the effect of the magnetic-fieldinduced confinement dominates.

All observed features, except for the $\mathrm{S}_{1}$, exhibit a diamagnetic shift (a blue shift) in magnetic field characteristic for an $s$-shell related emission. It means that their magnetic-field evolution is quadratic in the lowfield range. The $S_{1}$ energy experience a red shift with increasing field before it completely disappears from the spectrum at magnetic field of $2 \mathrm{~T}$. In our opinion, the $\mathrm{S}_{1}$ emission line is associated with a more complex configuration of the carriers and therefore further studies to explain its assignment are required.

\subsection{PLE spectrum of the neutral exciton}

A complicated character of the confining potential of the studied dots is also reflected in the PLE spectra of the neutral exciton - Fig. 3. In the used energy range of the excitation light, the PL spectra of the QD exhibit the emissions attributed to the transitions within the $p$-shell levels, displaying a more complex magnetic-field evolution that differs from the diamagnetic shift appearing for the $s$-shell emission. However, the PLE spectra of the neutral exciton display a series of resonances: $R_{1}, R_{2}, \ldots$ describing the $s$-shell behaviour in the magnetic field (see Fig. 3a). 

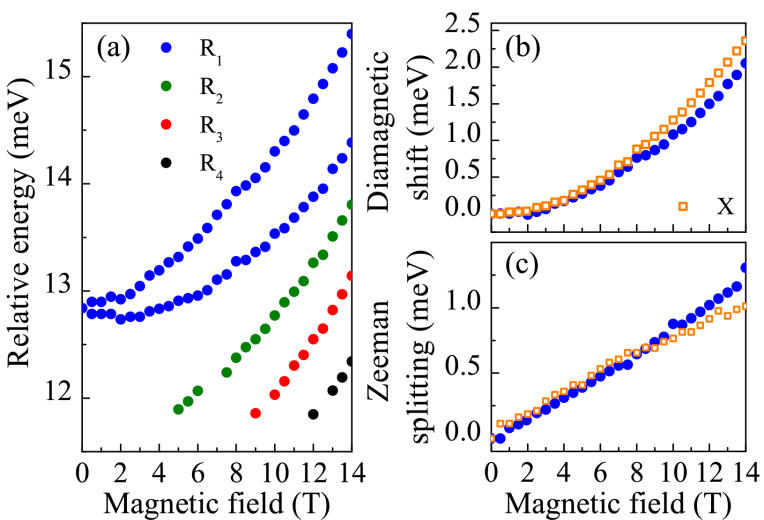

Fig. 3. (a) The evolution of the resonances of the neutral exciton as a function of magnetic field. The energy axis is rescaled with reference to the $\mathrm{X}$ energy at zero magnetic field. (b) The diamagnetic shift and (c) the Zeeman splitting of the $\mathrm{X}$ line and the $\mathrm{R}_{1}$ resonance.

In this paragraph, we focus on studies of the $R_{1}$ resonance, due to the observation of its dependence in a whole range of magnetic field. The diamagnetic shift of the $R_{1}$ resonance is smaller than the value obtained for the $\mathrm{X}$ line, as it is presented in Fig. 3b. In the case of the Zeeman splitting - see Fig. 3c, up to approximately $9 \mathrm{~T}$, the splittings are comparable for the $\mathrm{R}_{1}$ resonance and the $\mathrm{X}$ line, but at higher fields the difference becomes more pronounced. It excludes the participation of a phonon in the carriers relaxation processes, which lead to this resonance. The observation of the $s$-shell like resonances might be attributed to the transition between the excited hole levels, e.g. the $p-, d-, \ldots$ shells, and the ground electron level, the $s$-shell [4]. It is possibly related to the effect of some symmetry breaking, as a result of mixing with higher energy bands [15]. The analysis of the PLE spectra of the neutral exciton measured on a set of single QDs at $0 \mathrm{~T}$ leads to the conclusion that the energy separation between the $s$-shell-like resonances equals $\approx 1-2 \mathrm{meV}$. Because of the presented origin of resonances as a process involving consecutive excited hole levels, we can state that in the investigated QDs the energy separation between hole levels is only around a few $\mathrm{meV}$. At the same time, the observed energy distance between the $s$ - and $p$-shell emission in the PL spectra (around $13 \mathrm{meV}$ ) is mainly related to the energy separation between the electron levels.

\section{Conclusions}

In conclusion, the optical properties of a single GaAlAs/AlAs QD have been investigated using a PL and a PLE spectroscopy. It has been shown that the non-resonant and resonant excitation regimes involve two different mechanisms of the carrier trapping in the dot, which substantially affects the PL spectra. The resonances occurring in the PLE spectrum of the neutral exciton follow the magnetic-field evolution characteristic of the $s$-shell in a QD. In our opinion, the origin of the resonance may be related to the transitions between the excited hole levels in the valence band and the ground electron level in the conduction band. We have found that in the studied QDs the energy separation between consecutive levels in the hole ladder is only around a few $\mathrm{meV}$.

\section{Acknowledgments}

The work has been supported by the Foundation for Polish Science International Ph.D. Projects Programme co-financed by the EU European Regional Development Fund and by EuroMagNETII (JRA8) under the EU contract 228043. M.M. kindly acknowledges the National Science Center (decisions DEC-2013/08/T/ST3/00665 and DEC-2013/09/N/ST3/04237) for financial support for his Ph.D.

\section{References}

[1] I.A. Akimov, K.V. Kavokin, A. Hundt, F. Henneberger, Phys. Rev. B 71, 075326 (2005).

[2] P. Michler, A. Kiraz, C. Becher, W.V. Schoenfeld, P.M. Petroff, L. Zhang, E. Hu, A. Imamoglu, Science 290, 2282 (2000).

[3] P. Hawrylak, G.A. Narvaez, M. Bayer, A. Forchel, Phys. Rev. Lett. 85, 389 (2000).

[4] Y. Benny, Y. Kodriano, E. Poem, S. Khatsevitch, D. Gershoni, P.M. Petroff, Phys. Rev. B 84, 075473 (2011).

[5] A. Wysmołek, B. Chwalisz, M. Potemski, R. Stępniewski, A. Babiński, S. Raymond, Acta Phys. Pol. A 106, 367 (2004).

[6] A. Trüby, M. Potemski, R. Planel, Solid-State Electron. 40, 139 (1996).

[7] M. Molas, K. Gołasa, B. Piętka, M. Potemski, A. Babiński, Acta Phys. Pol. A 122, 988 (2012).

[8] M.D. Martín, C. Antón, L. Vińa, B. Piętka, M. Potemski, Europhys. Lett. 100, 67006 (2012).

[9] B. Piętka, J. Suffczyński, M. Goryca, T. Kazimierczuk, A. Golnik, P. Kossacki, A. Wysmolek, J.A. Gaj, R. Stępniewski, M. Potemski, Phys. Rev. B 87, 035310 (2013).

[10] A. Babiński, M. Potemski, S. Raymond, M. Korkusinski, W. Sheng, P. Hawrylak, Z. Wasilewski, Physica E 34, 288 (2006).

[11] M.R. Molas, A.A.L. Nicolet, M. Potemski, A. Babiński, Acta Phys. Pol. A 124, 785 (2013).

[12] W.-H. Chang, H.-S. Chang, W.-Y. Chen, T.M. Hsu, T.-P. Hsieh, J.-I. Chyi, N.-T. Yeh, Phys. Rev. B $\mathbf{7 2}$, 233302 (2005).

[13] T. Kazimierczuk, J. Suffczyński, A. Golnik, J.A. Gaj, P. Kossacki, P. Wojnar, Phys. Rev. B 79, 153301 (2009).

[14] T. Kazimierczuk, T. Smoleński, M. Goryca, Ł. Kłopotowski, P. Wojnar, K. Fronc, A. Golnik, M. Nawrocki, J.A. Gaj, P. Kossacki, Phys. Rev. B 84, 165319 (2011).

[15] T. Warming, E. Siebert, A. Schliwa, E. Stock, R. Zimmermann, D. Bimberg, Phys. Rev. B 79, 125316 (2009). 